\title{
Satellite Observations Show Reductions in Light Emissions at International Dark Sky Places During 2012-2020
}

\author{
Christopher C.M. Kyba ${ }^{1, *}$, Jacqueline Coesfeld ${ }^{1}$ \\ ${ }^{1}$ GFZ German Research Centre for Geosciences, 14473 Potsdam, Germany
}

Received 14 April 2021, Accepted 05 July 2021

\begin{abstract}
Hyde et al. previously examined the trends in light emission measured by satellite for 98 communities located in or near areas certified as "International Dark Sky Places" (IDSP), and did not find evidence of a difference in trends in comparison to 98 communities of similar size located further away. Here we re-examine the satellite dataset, making use of a newly available correction for the radiance of atmospheric airglow, and extending the analysis period by an additional two years. The new dataset is consistent with the hypothesis that light emissions tend to decrease in communities in or near certified IDSP (median value of $-1.6 \%$ per year), and is in tension with the hypothesis that there is no difference between these communities and others located further away (median increase of $+0.1 \%$ per year). While the null hypothesis of no difference in the certified regions still cannot be entirely ruled out (Kolmogorov-Smirnov test probability of 2.5\%), it appears likely that IDSP certification is associated with changes in light emissions.
\end{abstract}

Keywords: International Dark Sky Places, remote sensing, VIIRS DNB, nighttime lights, light pollution

\section{Introduction}

The "International Dark Sky Places" (IDSP) program run by the International Dark-Sky Association seeks "to encourage communities, parks and protected areas around the world to preserve and protect dark sites through responsible lighting policies and public education" [1]. In a recent article, Hyde et al. used satellite data to examine whether IDSP certification affects trends in light emissions. They compared trends in light emissions in communities in selected IDSP in Europe and North America from 2012-2018 with those for similarly sized communities outside of IDSP [2]. While several IDSP showed reductions in lighting compared to their partner regions, overall, Hyde et al. concluded that the data were also consistent with there being no differences between communities in IDSP and communities outside. We have since learned that the data for 2017 and 2018 were affected by a change in NASA's calibration of the satellite sensor [3]. This change in calibration caused the radiance reported for regions of Earth without any installed lighting to increase in 2017 and 2018 compared to the 2012-2016 period. In this article, we re-examine these communities using a newly developed correction that reduces the noise in the satellite data [4], and including additional data from 2019 and 2020.

The overall approach here is very similar to Hyde et al. [2], and we therefore suggest readers interested in more detailed background information about the IDSP program and the satellite sensor (the Visible Infrared Imaging Radiometer Suite Day-Night Band; DNB) review that paper before continuing further here. Briefly, areas that are certified as IDSP have intentionally committed to reducing existing light emissions, minimizing the environmental impact of future emissions, and educating visitors about the value of sustainable lighting. The DNB is a satellite radiometer that measures the radiance of Earth at night in the band 500-900 nm [5], for which monthly composite

${ }^{*} \mathrm{C}$ Kyba, Email address: kyba@gfz-potsdam.de 
images are built up of data on cloud and moon-free nights by the Earth Observation Group [6]. Hyde et al. formulated four hypotheses to test [2]:

H1) Annual changes in light emissions from towns in or near IDSP are smaller (or more negative) than the changes in similar towns that are located further away from IDSP.

H2) Towns in or near IDSP experience an absolute reduction in light emissions during a time period overlapping the certification.

H3) The distribution of annual changes in light emissions for communities in or near IDSP is not the same as the distribution for communities further away from IDSP.

H4) The distribution of annual trends in light emission changes for the sample of communities tested is not normally distributed.

Hyde et al. found that the first two hypotheses could not be effectively tested by the data, because the trends in light emissions from IDSP and partners were too similar (i.e. H3 was not observed). The fourth hypothesis was found to be incorrect, as the distribution in growth per year was normally distributed for both sets of communities.

Here, we re-examine the first three hypotheses using monthly DNB datasets that have been corrected to center the radiance of unlit regions at zero [4,7]. The correction is derived by observing regions known to have no human settlements, and then interpolating to correct positions between these regions. This prevents unlit areas from incorrectly appearing to have an increase in radiance, as is the case for the uncorrected images. In addition, the correction reduces the noise in the dataset (due to variations in nighttime atmospheric airglow), and the effective sensitivity of the DNB is thereby increased for regions with small amounts of artificial light emission. Readers interested in more details about the correction are referred to Coesfeld et al. [4].

\section{Methods}

As in Hyde et al. [2], we compare 98 communities inside of IDSP boundaries with 98 communities of similar size but located 100-300 km away within the same country. For convenience, details about the IDSP and their positions are reproduced from Hyde et al. [2] in Tables 1 and 2, as well as Figure 1. Two different types of IDSP were examined, International Dark Sky Reserves (IDSR), and International Dark Sky Communities (IDSC). As explained in Hyde et al. [2] "The IDSRs are large regions, and typically contain numerous small towns. The IDSCs are individual communities, and are often incorporated municipalities. In both cases, certification requires the communities to voluntarily commit to transition to sustainable lighting practice." All IDSP were certified in either 2014 or 2015, meaning the satellite data record has at least two years of data before certification. A considerably larger number of communities were analyzed for IDSR Rhön than for the other categories, and this set of communities was further divided into two groups, according to the status of lighting legislation in each community at the time that Hyde et al. was published. In this paper, we use the identical set of communities and groupings. Boundaries for the lit areas of the communities were drawn using the "Radiance Light Trends" web application [8], and are available online [9].

Table 1: International Dark Sky Places included in the analysis. The asterisk (*) indicates that Silver Cliff and Westcliffe are designated as a single IDSC, but were analyzed as two separate areas. Table and text reproduced from[2].

\begin{tabular}{lccc}
\hline IDSP Type & IDSP Name & Year of certification & Size (km²) \\
\hline Reserve & Kerry & 2014 & 700 \\
Reserve & Rhön & 2014 & 1720 \\
Reserve & Snowdonia National Park & 2015 & 2132 \\
Reserve & Westhavelland Nature Park & 2014 & 748 \\
Community (suburban) & Beverly Shores & 2014 & 15 \\
Community (suburban) & Bon Accord & 2015 & 2.1 \\
Community (exurban) & Dripping Springs & 2014 & 303 \\
Community (exurban) & Horseshoe Bay & 2015 & 30 \\
Community (rural) & Kaibab Paiute Indian & 2015 & 489 \\
Community (rural) & Reservation & 2014 & 50 \\
Community (rural) & Sedona & 2015 & $43^{*}$ \\
\hline
\end{tabular}


Table 2: Groupings used for the analysis. Table and text reproduced from [2].

\begin{tabular}{lccc}
\hline IDSP Type & Grouping & Total \# communities & Total \# comparison towns \\
\hline Reserve & Kerry, Snowdonia, Westhavelland & 14 & 14 \\
Reserve & All of Rhön & 76 & 76 \\
Reserve & Rhön legislation & 39 & 39 \\
Reserve & Rhön no legislation & 37 & 37 \\
Community & All IDSC & 8 & 8 \\
Combined & All towns in IDSR and IDSC & 98 & 98 \\
\hline
\end{tabular}

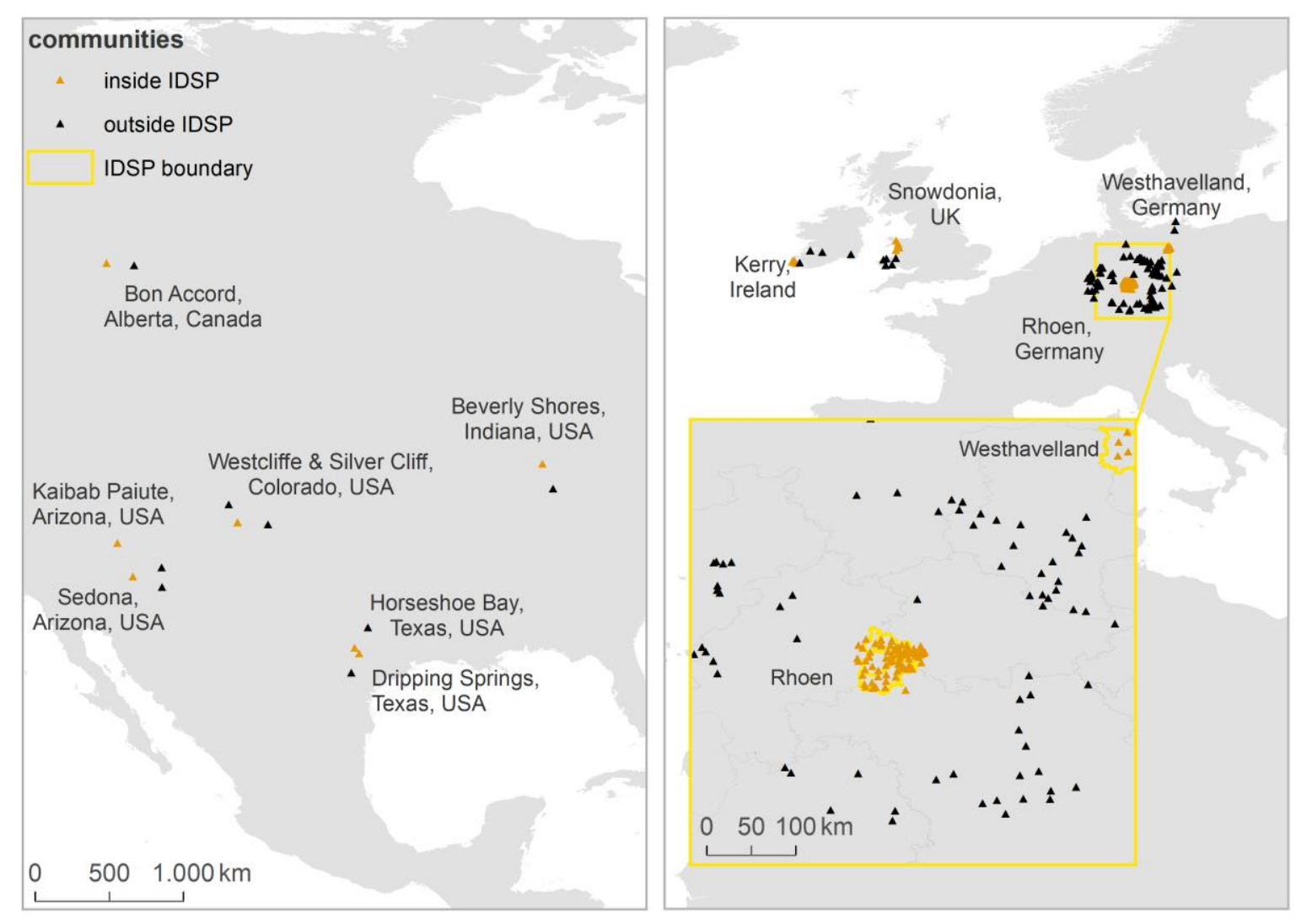

Figure 1: Map showing the locations of the examined communities. Communities inside of IDSP are indicated with an orange triangle, partner communities with a black triangle. The boundaries of IDSR Rhön and Westhavelland are shown in the inset. The background country maps are based on GADM version 2.6 (2018), https://gadm.org/download_world.html. Figure and text reproduced from [2].

Nighttime light emissions in the 500-900 $\mathrm{nm}$ band were obtained by the Visible Infrared Imaging Radiometer Suite Day-Night Band radiometer [5]. Monthly cloud- and moon-free composites were obtained from the Earth Observation Group [6, https://eogdata.mines.edu/download_dnb_composites.html], and corrected to reduce the influence of atmospheric airglow [4,7]. To reduce variability due to seasonal changes (and especially snow cover), we analyzed only the months of September, October, and November (as in Hyde et al. [2]). These months are generally the most stable in the northern hemisphere. (Data is not available at mid latitudes in the summer months, because the elevation of the sun is high enough that the land is in twilight, and in some cases shines stray light on the satellite sensor. We do not use "stray light corrected data", as this has the potential to introduce noise at our generally dark sites.) For some IDSP at high latitudes, stray light on the satellite meant that even data in September was not available. In these cases, we simply did not include a data point for that month.

Hyde et al. [2] reported the results of an exponential fit to the data from within the Radiance Light Trends application. Here, we instead analyzed the data using python scripts. Day/Night Band pixels were included in the analysis if the pixel center was included within the area defined by the polygon. In a small number of cases (22 out of 4728 data points), the radiance observed from a community was reported as negative, due to the low intrinsic 
radiance and natural variability in airglow. While this is to be expected, it introduces a problem for doing a weighted least squares exponential fit. The reason for this is that the fit uses the logarithm of the value, which is undefined for values less than or equal to zero. Since removing negative data points would result in a bias (positive fluctuations are included, but negative ones or not), we instead set the mean radiance in these cases to a small positive value $\left(0.001 \mathrm{nW} / \mathrm{cm}^{2} \mathrm{sr}\right)$. Based on our experience, this is small enough to be basically equivalent to no artificial light, while large enough to avoid having an undue influence on the fit. The fit was performed in python using the NumPy polyfit routine, which gives identical results to those from Radiance Light Trends for identical data.

As noted by Hyde et al. [2], the data from the different IDSP are not independent observations, due to the nearness of the communities (meaning they are viewed from similar angles and through similar atmospheres). Furthermore, the new airglow correction has a similar value for locations at short distances from each other [4]. This results in correlations in the radiance data [10], but with an unknown degree. These correlations mean that the results of standard statistical tests are not valid. Nevertheless, as was the case in Hyde et al. [4], it is still possible to check at least whether the results appear to be consistent with the hypotheses or not.

\section{Results and discussion}

Applying the airglow correction (which shifts areas with no light emission to have an average of zero radiance in the DNB data) had a considerable impact on the results compared to those presented in Hyde et al. [2]. Whereas Hyde et al. concluded that the impact of certification on radiance was ambiguous, in the present analysis the results are consistent with certification having an impact on lighting trends (Table 3). In the case of the IDSC and three of the four IDSR, the towns inside of a certified area had a median annual decrease in light, while the similarly sized towns in areas with no certification had no change, or a median annual increase. The only exception was IDSR Snowdonia, where decreases were observed both inside and outside of the IDSR. However, even in this case, the decreases were larger for the communities inside than for those outside. When all of the data is combined, settlements in areas with certification decreased light output by a median rate of $1.6 \%$ per year, while settlements in areas without certification increased light output at a median rate of $0.1 \%$ per year. The results are therefore consistent with both hypothesis 2 (that certification should lead to decreases in light emission) and hypothesis 1 (that decreases should be larger in areas that are certified).

Table 3: Summary of light trends results. The average changes during the period 2012-2020 are shown, along with the standard deviation. The four International Dark Sky Reserves are shown separately, and the "combined" group is the combination of Westhavelland, Snowdonia, and Kerry. The "total" group includes towns from all International Dark Sky Places combined. For Rhön, "approved" indicates that the community had approved legislation restricting light list at the time Hyde et al. [2] was published.

\begin{tabular}{|c|c|c|c|c|c|}
\hline Name & Class & $\begin{array}{l}\text { Number of } \\
\text { towns }\end{array}$ & $\begin{array}{c}\text { Median annual change } \\
(\%)\end{array}$ & $\begin{array}{c}\text { Mean annual } \\
\text { change }(\%)\end{array}$ & $\begin{array}{c}\text { Standard } \\
\text { deviation }(\%)\end{array}$ \\
\hline \multirow{4}{*}{ Rhön } & Inside & 76 & -0.7 & -0.6 & 6.2 \\
\hline & Outside & 76 & 0.0 & 0.0 & 5.1 \\
\hline & Approved & 39 & +0.2 & -0.6 & 6.4 \\
\hline & Not approved & 37 & -2.4 & -0.6 & 6.0 \\
\hline \multirow{2}{*}{ Kerry } & Inside & 4 & -1.9 & -1.7 & 1.0 \\
\hline & Outside & 4 & +1.8 & +1.8 & 3.1 \\
\hline \multirow{2}{*}{ Snowdonia } & Inside & 5 & -6.8 & -7.8 & 2.3 \\
\hline & Outside & 5 & -3.5 & -4.0 & 2.0 \\
\hline \multirow{2}{*}{ Westhavelland } & Inside & 5 & -2.1 & -2.0 & 2.1 \\
\hline & Outside & 5 & +2.3 & +2.5 & 1.2 \\
\hline \multirow{2}{*}{ IDSR combined } & Inside & 14 & -2.8 & -3.9 & 3.4 \\
\hline & Outside & 14 & -0.1 & 0.0 & 3.7 \\
\hline \multirow{2}{*}{ Communities } & IDSC & 8 & -1.0 & -1.3 & 2.8 \\
\hline & Not IDSC & 8 & +1.0 & +0.7 & 3.2 \\
\hline \multirow{2}{*}{ Total } & IDSP & 98 & -1.6 & -1.1 & 5.8 \\
\hline & Not IDSP & 98 & +0.1 & +0.1 & 4.8 \\
\hline
\end{tabular}


However, while the data are consistent with the first two hypotheses, it would be premature to entirely rule out the null hypothesis (that there is effectively no difference between the partner towns). The reason for this is that the standard deviation of the rates of change among the towns is quite large compared to the difference in the median changes (Table 3). The best fit for annual change in light emissions in many of the individual towns in regions with certification, for example, was for increasing light emissions (Figure 2). We computed the Kolmogorov-Smirnov (KS) statistic (using ks_2samp from scipy) for the two corrected distributions (shown in the right hand plot in Figure 2). The KS statistic was 0.19 , and the probability of observing a KS statistic this large by random chance is $2.5 \%$. The data are therefore consistent with hypothesis 3 (that there is a difference between the two distributions), but the null hypothesis (that there is no difference between the two distributions) is not yet decisively ruled out.
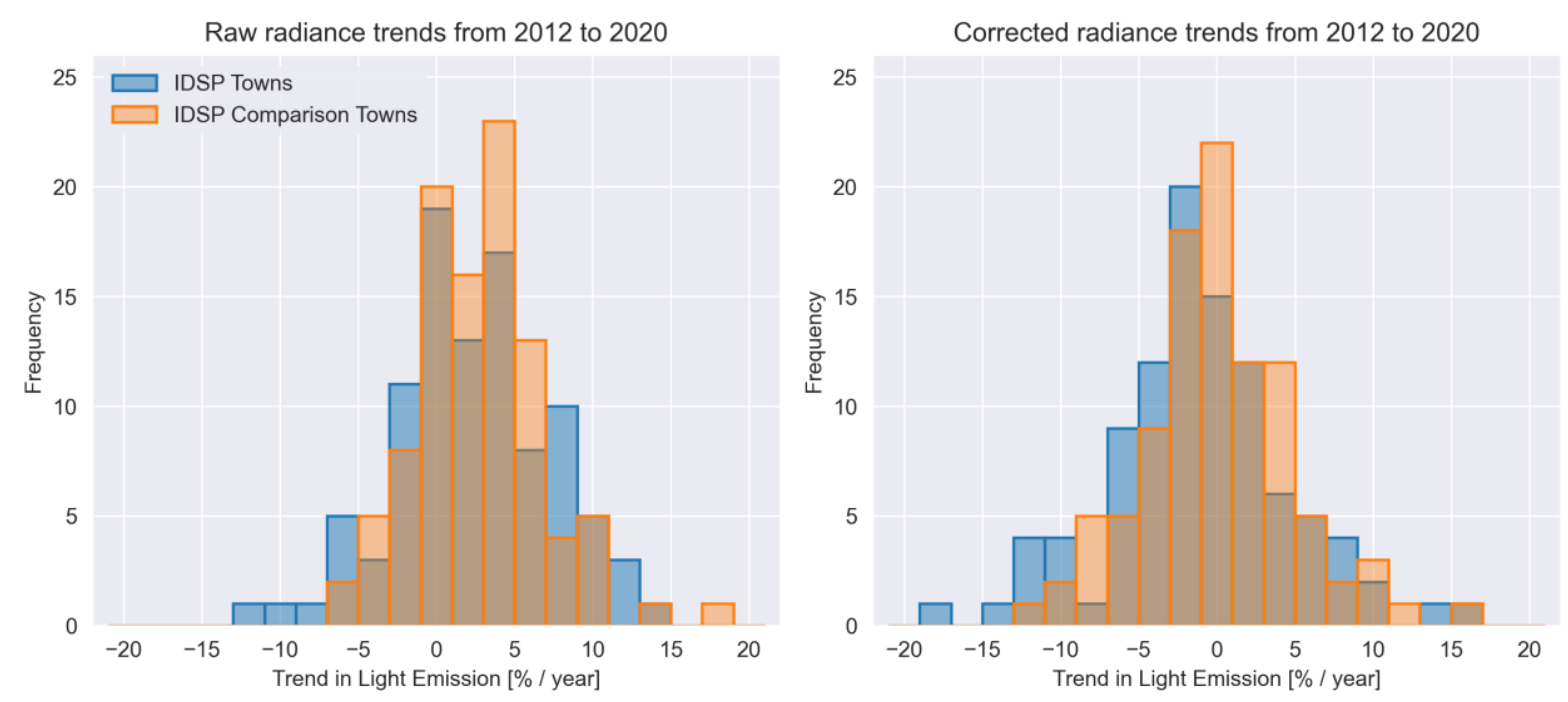

Figure 2: Histograms of fractional annual change in light emissions for all towns in or near IDSP and all partner towns. The data is shown twice, first with the uncorrected monthly composites (left), and second after applying the correction that sets unlit areas to zero radiance (right). The data in Table 3 is based on the right (corrected radiance) panel.

These data also demonstrate the value of the airglow correction for DNB data [4,7], at least in areas with small amounts of lighting. The differences between the two sets of distributions are more apparent once the correction is applied (Figure 2). In addition, the center of the distributions shift noticeably downward in both cases - the overall increase in the left panel (and presumably the increases previously reported in Hyde et al. [2]) are an artifact due to the change in calibration of DNB data in 2017. This is even more apparent when the results that would have been obtained for the 2012-2020 dataset if the correction was not applied (Table A1) are compared to the corrected results shown in Table 3.

\section{Discussion and conclusions}

The 98 communities inside or near to areas certified as International Dark Sky Places are characterized by an overall decrease in light emissions (in the 500-900 $\mathrm{nm}$ band) during 2012-2020 (-1.1\% per year), with individual communities frequently having considerably larger or smaller values (standard deviation of 5.8\%). This compares to an average increase of $0.1 \%$ per year for 98 similarly sized communities in the same countries, and to an average increase of $2.2 \%$ per year globally [11]. The distributions in trends in light emissions between IDSP and partner towns are distinct enough that the probability that they are drawn from the same distribution (evaluated by a KS test) is quite small. However, given the considerable variation in DNB data due to atmospheric and other factors $[10,12]$, these data are not independent observations. We therefore conclude that it is appropriate to be cautiously optimistic regarding the impact of certification, but that it is still premature to make any overly strong claims. This is especially the case given that conversion of outdoor lighting from high pressure sodium to white LED while maintaining luminous flux could lead to an apparent decrease in radiance observed by the DNB instrument emissions [13]. Continued observation of light emissions from these communities, and the examination of a larger 
set of communities should provide stronger evidence for an impact if it is really the case that certification results in decreases in light emissions.

In regions with small amounts of light emissions, it is critically important to correct the DNB time series for variability in airglow emissions, and in particular the step caused by the change in NASA's calibration of the DNB sensor. The application of an airglow correction [4] led to a much stronger conclusion here than in our original analysis (Hyde et al. [2]), and the average increases in light emissions for IDSP reported in Hyde et al. were likely an artifact due to NASA's calibration change. This is supported by the data in Table A1, which shows that an analysis that did not correct for the change in calibration would incorrectly report increases in many communities, analogous to what was shown for the complete land area of North Korea in Coesfeld et al. [4].

Finally, we wish to conclude by restating the final conclusion of Hyde et al. [2]: Satellite datasets measure upward emission, not sky brightness. Understanding changes in night sky radiance in the IDSP therefore requires ground based observations [14]. Satellite datasets provide useful complementary information, particularly in regards to the installation of new lighting or the elimination of unnecessary lighting. However, the different observation directions mean that satellite datasets can most likely never eliminate the need for ground observations to understand changes in night sky brightness.

\section{Acknowledgements}

We acknowledge the funding received through the European Union's Horizon 2020 research and innovation programme ERA-PLANET, grant agreement no. 689443, via the GEOEssential project, and funding from the Helmholtz Association Initiative and Networking Fund under grants ERC-RA-0031 and CS-0003. Image and Data processing by the Earth Observation Group, Payne Institute for Public Policy, and formerly by NOAA's National Geophysical Data Center. We salute the communities and parks examined in this project for their work in achieving IDSP certification, and for their commitment towards the principle of sustainable lighting.

\section{References}

[1] International Dark-Sky Association. (n.d). International Dark Sky Places Program. Retrieved October 15, 2020, from https://www.darksky.org/ our-work/conservation/idsp/.

[2] Hyde, E., Frank, S., Barentine, J. C., Kuechly, H., \& Kyba, C. C. (2019). Testing for changes in light emissions from certified International Dark Sky Places. International Journal of Sustainable Lighting, 21(1), 11-19.

[3] Uprety, S., Cao, C., Gu, Y., \& Shao, X. (2017, July). Improving the low light radiance calibration of S-NPP VIIRS Day/Night Band in the NOAA operations. In 2017 IEEE International Geoscience and Remote Sensing Symposium (IGARSS) (pp. 4726-4729). IEEE.

[4] Coesfeld, J., Kuester, T., Kuechly, H. U., \& Kyba, C. (2020). Reducing Variability and Removing Natural Light from Nighttime Satellite Imagery: A Case Study Using the VIIRS DNB. Sensors, 20(11), 3287.

[5] Miller, S. D., Straka, W., Mills, S. P., Elvidge, C. D., Lee, T. F., Solbrig, J., ... \& Weiss, S. C. (2013). Illuminating the capabilities of the suomi national polar-orbiting partnership (NPP) visible infrared imaging radiometer suite (VIIRS) day/night band. Remote Sensing, 5(12), 6717-6766.

[6] Elvidge, C. D., Baugh, K. E., Zhizhin, M., \& Hsu, F. C. (2013). Why VIIRS data are superior to DMSP for mapping nighttime lights. Proceedings of the Asia-Pacific Advanced Network, 35(0), 62.

[7] Coesfeld, Jacqueline; Kuester, Theres; Kuechly, Helga U.; Kyba, Christopher C.M. (2020): Background correction for EOG VIIRS-DNB monthly composites (data and scripts). GFZ Data Services. https://doi.org/10.5880/GFZ.1.4.2020.003

[8] Stare, J., \& Kyba, C. (2019). Radiance Light Trends.

[9] Hyde, E., \& Kyba, C. (2019). Analysis boundaries and lighting trends (2012-2018) for selected International Dark Sky Places.

[10] Coesfeld, J., Anderson, S. J., Baugh, K., Elvidge, C. D., Schernthanner, H., \& Kyba, C. (2018). Variation of individual location radiance in VIIRS DNB monthly composite images. Remote Sensing, 10(12), 1964.

[11] Kyba, C. C., Kuester, T., De Miguel, A. S., Baugh, K., Jechow, A., Hölker, F., ... \& Guanter, L. (2017). Artificially lit surface of Earth at night increasing in radiance and extent. Science advances, 3(11), e1701528.

[12] Levin, N., \& Zhang, Q. (2017). A global analysis of factors controlling VIIRS nighttime light levels from densely populated areas. Remote sensing of environment, 190, 366-382.

[13] Levin, N., Kyba, C. C., Zhang, Q., de Miguel, A. S., Román, M. O., Li, X., ... \& Elvidge, C. D. (2020). Remote sensing of night lights: A review and an outlook for the future. Remote Sensing of Environment, 237, 
111443.

[14]Barentine, J. C. (2019). Methods for assessment and monitoring of light pollution around ecologically sensit ive sites. Journal of Imaging, 5(5), 54.

\section{Appendix}

Table A1. Summary of raw (uncorrected) light trends results. The average changes during the period 2012-2020 are shown, along with the standard deviation. The four International Dark Sky Reserves are shown separately, and the "combined" group is the combination of Westhavelland, Snowdonia, and Kerry. The "total" group includes towns from all International Dark Sky Places combined. For Rhön, "approved" indicates that the community had approved legislation restricting light list at the time Hyde et al. [2] was published.

\begin{tabular}{|c|c|c|c|c|c|}
\hline Name & Class & $\begin{array}{c}\text { Number of } \\
\text { towns }\end{array}$ & $\begin{array}{c}\text { Median annual change } \\
(\%)\end{array}$ & $\begin{array}{c}\text { Mean annual } \\
\text { change }(\%)\end{array}$ & $\begin{array}{c}\text { Standard } \\
\text { deviation }(\%)\end{array}$ \\
\hline \multirow{4}{*}{ Rhön } & Inside & 76 & +3.3 & +3.4 & 4.8 \\
\hline & Outside & 76 & +3.1 & +3.1 & 4.1 \\
\hline & Approved & 39 & +2.5 & +2.8 & 5.0 \\
\hline & Not approved & 37 & +4.0 & +4.1 & 4.6 \\
\hline \multirow{2}{*}{ Kerry } & Inside & 4 & -0.2 & -0.1 & 1.4 \\
\hline & Outside & 4 & +2.4 & +2.7 & 2.7 \\
\hline \multirow{2}{*}{ Snowdonia } & Inside & 5 & -6.3 & -6.5 & 1.7 \\
\hline & Outside & 5 & -2.2 & -2.5 & 2.0 \\
\hline \multirow{2}{*}{ Westhavelland } & Inside & 5 & +0.4 & +0.6 & 1.9 \\
\hline & Outside & 5 & +4.8 & +4.9 & 1.5 \\
\hline \multirow{2}{*}{ IDSR combined } & Inside & 14 & -1.5 & -2.2 & 3.7 \\
\hline & Outside & 14 & +1.4 & +1.6 & 3.8 \\
\hline \multirow{2}{*}{ Communities } & IDSC & 8 & +0.3 & +0.3 & 1.6 \\
\hline & Not IDSC & 8 & +1.7 & +1.4 & 3.1 \\
\hline \multirow{2}{*}{ Total } & IDSP & 98 & +1.9 & +2.4 & 4.9 \\
\hline & Not IDSP & 98 & +2.8 & +2.8 & 4.0 \\
\hline
\end{tabular}

